\title{
ON A SEQUENCE OF ALMOST DETERMINISTIC PAIRWISE INDEPENDENT RANDOM VARIABLES
}

\author{
A. JOFFE
}

Abstract. We show the existence of infinite sequences of pairwise independent random variables (which are not constant almost everywhere) such that the whole sequence is determined with probability one by the knowledge of any pair of members of that sequence.

1. Introduction. We want to show the existence of infinite sequences $Y_{1}, Y_{2}, \cdots, Y_{n}, \cdots$ of pairwise independent random variables (which are not constant almost everywhere) having the property that for any $i \neq j$ there exist Borel functions $g_{n}$ such that

$$
Y_{n}=g_{i, j, n}\left(Y_{i}, Y_{j}\right), \quad n=1,2,3, \cdots .
$$

This answers a question raised by D. Dawson. For finite sets, such a construction is done in [3].

2. Construction. We will first construct a sequence of random elements having the properties mentioned in the introduction. Let $G$ be the character group of $Q$, the additive group of the rational numbers with the discrete topology. $G$ is an abelian compact group which is divisible and torsion free. Moreover $G$ is metrizable, complete and separable. There is a lot of information in the literature about $G$, but we refer only to [2] where one can find other references. $G$ being compact, the Haar measure $\mu$ is uniquely determined on the Borel sets of $G$ by $\mu(G)=1$. Take as probability space $\Omega=G \times G$ the direct product of the group $G$ with itself, and for measure on $\Omega$ take the Haar measure $\mu \times \mu$ which is the product measure of $\mu$ with itself. Let $X_{1}$ (resp. $X_{2}$ ) denote the projection maps of $G \times G$ onto the first (resp. second) coordinates. $X_{1}$ and $X_{2}$ are random independent elements taking values in $G$. Define

$$
X_{n}=(n-2) X_{1}+X_{2}, \quad n=2,3, \cdots .
$$

Given any $i \neq j$ it is easily verified, since $G$ is a vector space over the rationals, that the relations given by (1) define $X_{1}$ and $X_{2}$ uniquely in terms of $X_{i}$ and $X_{j}$; moreover if we write $\left(X_{1}, X_{2}\right)=g_{i, j}\left(X_{i}, X_{j}\right), g_{i, j}$

Received by the editors September 14, 1970.

AMS 1970 subject classifications. Primary 60A05; Secondary 60B15.

Key words and phrases. Pairwise independent random variables, abelian compact group, divisible torsion free groups.

Copyright (c) 1971, American Mathematical Society 
is an automorphism of $\Omega$. Let us compute the image $\tilde{\mu}$ of $\mu$ under this automorphism. For any set $A \times B$ of $\Omega$ where $A$ and $B$ are Borel sets of $G$ we have

$$
\begin{aligned}
\tilde{\mu}(A \times B) & =\mu \times \mu\left\{\left(X_{i}, X_{j}\right) \in A \times B\right\} \\
& =\mu \times \mu\left\{\left(X_{i}, X_{j}\right) \in g_{i, j}^{-1}(A \times B)\right\} .
\end{aligned}
$$

Since $g_{i, j}$ is a group isomorphism it follows that $\tilde{\mu}(A \times B)$ is translation-invariant and therefore $\tilde{\mu}$ is a Haar measure; since $g$ is onto $\tilde{\mu}(G \times G)=1$ and $\tilde{\mu}=\mu \times \mu$. It follows that

$$
\mu \times \mu\left\{\left(X_{i}, X_{j}\right) \in A \times B\right\}=\mu\left(X_{i} \in A\right) \cdot \mu\left(X_{j} \in B\right)=\mu(A) \cdot \mu(B) .
$$

Our sequence $X_{1}, X_{2}, \cdots, X_{n}, \cdots$ is a sequence of random elements having the properties stated in the introduction. To establish the existence of random variables having these properties, it suffices to note that $G$ being complete and separable there is a Borel isomorphism $\phi$ of $G$ on $[0,1]$ where the image of $\mu$ is Lebesgue measure. See for instance $\left[1\right.$, p. 339]. If we define $Y_{n}=\phi\left(X_{n}\right)$ we have established the following

Theorem. The sequence $Y_{1}, \cdots, Y_{n}, \cdots$ defines pairwise independent random variables. Each of the $Y_{i}$ is uniformly distributed on $[0,1]$ and for each $i, j, i \neq j$, there are Borel functions $g_{i, j, n}$ such that $Y_{n}$ $=g_{i, j, n}\left(Y_{i}, Y_{j}\right), n=1,2, \cdots$.

3. Acknowledgement. This work would never have been done without many cups of coffee absorbed with many colleagues at Mc Gill or at the Fleisher Foundation. ${ }^{1}$ These conversations introduced the author to various topics in algebra and harmonic analysis, many of which were essential to the above construction.

\section{REFERENCES}

1. P. Halmos and J. von Neumann, Operator methods in classical mechanics. II, Ann. of Math. (2) 43 (1942), 332-350. MR 4, 14.

2. E. Hewitt and K. Ross, Abstract harmonic analysis. Vol. 1: Structure of topological groups. Integration theory, group representations, Die Grundlehren der math. Wissenschaften, Band 115, Academic Press, New York; Springer-Verlag, Berlin, 1963. MR 28 \#158.

3. A. Joffe, On a set of almost deterministic pairwise independent random variables, Ann. Math. Statist. (to appear).

McGill University, Montreal, Quebec, Canada

Université de Montréal, Montréal, Québec, Canada

'Located for the time being at the "Auberge Joffe", Outremont, Canada. 